Revue musicale OICRM

\title{
Les sites Internet de compositeur-rices, ou la permanence des modèles d'écriture de soi
}

\section{Irina Kirchberg et Federico Lazzaro}

Volume 7, numéro 1, 2020

Écrits de compositeurs et espaces médiatiques

URI : https://id.erudit.org/iderudit/1069474ar

DOI : https://doi.org/10.7202/1069474ar

Aller au sommaire du numéro

\section{Éditeur(s)}

Observatoire interdisciplinaire de création et recherche en musique (OICRM)

ISSN

2368-7061 (numérique)

Découvrir la revue

Citer cet article

Kirchberg, I. \& Lazzaro, F. (2020). Les sites Internet de compositeur.rices, ou la permanence des modèles d'écriture de soi. Revue musicale OICRM, 7(1),

108-133. https://doi.org/10.7202/1069474ar
Résumé de l'article

En tant que production non musicale destinée à la lecture, le site Internet personnel d'un·e compositeur-rice peut être considéré, dans son ensemble, comme un " écrit » de compositeur en plus de servir d'archive pour différentes formes d'écrits par ou sur l'artiste (textes de présentation d'oeuvres, interviews, etc.). Les analyses comparées d'un type de texte omniprésent sur les sites de compositeur.rices - la biographie - ainsi que de l'approche au genre du texte de présentation d'une oeuvre permettent de considérer les stratégies d'autoprésentation et de présentation de leur musique mises en oeuvre par les compositeur.rices. Ces analyses aboutissent au constat d'une tendance générale à la production de contenus plutôt destinés aux professionnels de la musique et à la sous-exploitation des possibilités offertes par le numérique pour la promotion et la médiation de la musique contemporaine. 


\title{
Les sites Internet de compositeur.rices, ou la permanence des modèles d'écriture de soi
}

\author{
Irina Kirchberg \\ et Federico Lazzaro
}

\begin{abstract}
Résumé
En tant que production non musicale destinée à la lecture, le site Internet personnel d'un e compositeur rice peut être considéré, dans son ensemble, comme un " écrit " de compositeur en plus de servir d'archive pour différentes formes d'écrits par ou sur l'artiste (textes de présentation d'œuvres, interviews, etc.). Les analyses comparées d'un type de texte omniprésent sur les sites de compositeur rices - la biographie - ainsi que de l'approche au genre du texte de présentation d'une œuvre permettent de considérer les stratégies d'autoprésentation et de présentation de leur musique mises en œuvre par les compositeur-rices. Ces analyses aboutissent au constat d'une tendance générale à la production de contenus plutôt destinés aux professionnels de la musique et à la sous-exploitation des possibilités offertes par le numérique pour la promotion et la médiation de la musique contemporaine.

Mots clés : Internet ; écrits de compositeur rices ; musique contemporaine ; révolution digitale; sites de compositeur rices.
\end{abstract}

\begin{abstract}
As a non-musical production intended for reading, a composer's personal website can be considered, as a whole, as a composer's "writing" in addition to serving as an archive for different forms of writing by or about the artist (presentation texts about works, interviews, etc.). Comparative analyses of a certain type of text that is omnipresent on composers' sites - the biography - as well as the approach to a presentation text about a work provide an insight into the composers' selfpresentation strategies and the way they present their music. These analyses lead to the observation of a general tendency towards the production of contents for music professionals and the under-exploitation of the possibilities offered by digital technology for the promotion and mediation of contemporary music.

Keywords: composers' websites; composers' writings; contemporary music; digital revolution; Internet.
\end{abstract}


À une époque où la présence sur le web est un véritable diktat, les compositeur rices ne font pas exception et se mettent en vitrine par le biais de sites personnels qui s'intègrent parfois à ce qui s'apparente à des portfolios numériques (page Facebook, comptes Twitter, Instagram, etc.). En quoi l'ère numérique modifie-t-elle la relation des compositeur rices à l'espace médiatique ? Les nouveaux médias numériques changent-ils les conditions selon lesquelles les compositeur-rices promeuvent leurs œuvres ? Et, en ce qui concerne plus spécifiquement les écrits de compositeur·rices, quelle est leur nature et leur place dans leurs sites Internet personnels?

Dans son ouvrage La révolution digitale dans la musique (2017), Harry Lehmann décrit ce qu'il analyse comme des formes de désinstitutionnalisation de la musique de création à l'ère numérique. Sa thèse est que les outils numériques permettent une démocratisation de la création et de la réception de la "Nouvelle musique » : plus besoin de conservatoires pour apprendre à composer (les compositeur-rices peuvent le faire en ligne), plus besoin de sociétés de concerts pour se faire jouer (ils et elles peuvent utiliser des orchestres virtuels), plus besoin d'éditeurs pour publier ses partitions (que chacun $e$ peut rendre facilement accessibles en ligne après les avoir écrites avec un logiciel de notation musicale). Dans cette " profonde désinstitutionnalisation de la musique contemporaine " (Lehmann 2017, p. 17), le a compositeur-rice serait donc de plus en plus faber fortunae suae, ce qui prolonge les positions avancées par la sociologie du travail artistique sur la démultiplication des tâches qui incombent désormais aux artistes (Menger 2002 ; Sinigaglia-Amadio et Sinigaglia 2017).

Alors que Lehmann se demande si pouvoir tout faire sur le web suffit à la « démocratisation ${ }^{1} »$ de la musique de création, il ne considère pas la question des stratégies de mise en visibilité déployées à l'attention du grand public (vulgarisation et médiation). Voit-on apparaître des stratégies qui s'éloignent du modèle traditionnel à destination des pairs et des institutions et qui exploitent les possibilités offertes par ce nouveau médium ? En comparant le régime traditionnel des "institutions fortes " (conservatoires, sociétés de concert, éditeurs) avec le nouveau régime des "institutions faibles " numériques, le philosophe allemand écrit que ces dernières " réagissent dès qu'un compositeur a su attirer l'attention sur lui, de quelque manière que ce soit " (Lehmann 2017, p. 122, nous soulignons). C'est sur ces manières (numériques) adoptées par les compositeur rices pour attirer l'attention sur eux que nous nous concentrons dans notre étude des sites Internet personnels de compositeur rices de musique de création. «Attirer l'attention » est considéré au sens large : mise en vitrine des œuvres et de leur diffusion, mais aussi stratégies de démarcation par rapport aux collègues, que ce soit sur le plan de la formation, de la poétique, de la technique. Est-ce que l'analyse des sites de compositeur-rices et des écrits qu'ils contiennent

1 Lehmann insiste sur cet aspect notamment dans les chapitres "L'économie de la culture " (p. 105-120) et « La critique musicale » (p. 197-210). Il discute brièvement du problème de la classification de l'abondance du matériel disponible en ligne et de la réflexion sur la musique, en affirmant que la mort des éditeurs ouvre une nouvelle vie pour la critique, puisque la nécessité d'une hiérarchisation de la production musicale se placerait non plus en amont (sélection des œuvres dignes d'être vendues), mais $a$ posteriori (voir aux p. 24-25). 
révèle des stratégies de présentation de soi qui témoignent de la tendance vers la "désinstitutionnalisation » thématisée par Lehmann?

Le site web se prête à être à la fois un outil de promotion et de médiation. Dans une optique promotionnelle, est-ce que les compositeur-rices utilisent les possibilités offertes par l'outil numérique pour favoriser une distinction de leur musique dans un contexte de diffusion qui est ou sera de moins en moins institutionnalisé (si l'on accepte la thèse de Lehmann)? Si la promotion agit surtout en relation aux pairs, la médiation numérique de la culture est, selon Dujol et Mercier, " une démarche visant à [...] favoriser l'accès organisé ou fortuit, l'appropriation et la dissémination de contenus à des fins de diffusion des savoirs et des savoir-faire "; elle repose sur des " dispositifs sociotechniques qui permettent une mise en contact, un échange et la constitution du sentiment de l'être ensemble dans un espace commun(s) " (Dujol et Mercier 2017, p. 55-56). Est-ce que ces artistes exploitent les possibilités du multimédia pour réduire la fracture entre leurs créations et les publics, qu'ils dénoncent depuis au moins un siècle et que font apparaître les enquêtes sociologiques (Menger 1986; Dorin 2018) ? Notre analyse révèle plutôt que les compositeur rices qui ont fait l'objet de notre étude n'utilisent pas les potentialités de l'écriture numérique et perpétuent les modèles traditionnellement employés sur le marché des musiques de création programmées par des institutions (marché de niche et importance accordée au jugement des pairs ; voir notamment Menger [1983]2001).

\section{LE SITE COMME ÉCRIT DE COMPOSITEUR·RICE}

Cet article s'inscrit dans les thèmes de recherche sur les écrits de compositeur rices promus par le projet Dicteco. Dans cette perspective, nous avons à la fois étudié la présence d' "écrits " (déjà parus ailleurs ou pas) dans les sites et considéré les sites même comme des formes d'écriture. Emmanuel Reibel a proposé cinq définitions " progressivement englobantes » de la notion d'écrits de compositeur-rices. La cinquième, la plus englobante, comprend "l'ensemble des textes et paratextes ou énoncés, publiés, rédigés ou retranscrits par un compositeur » (Reibel 2013, p. 126). La notion ne considère que le rapport entretenu entre le.la compositeur rice et le verbal («l'Autre de la musique par excellence»; ibid., p. 127). À une époque où l'expression du verbal intègre de plus en plus systématiquement des aspects visuels (émoticons), hypertextuels (liens) et multimédia (vidéos, musique), la catégorie d' "écrit » semble cependant s'élargir davantage et englober, dans le cas qui nous occupe, la sélection et la mise en récit que les compositeur rices font du contenu autre que musical qu'ils produisent. Bref, même dans les cas où le site d'un·e compositeur rice ne contient pas d'écrits au sens étroit, il est en soi une forme d'écriture.

Le corpus

Notre étude interroge, d'une part, la nature des sites, leur forme et leur contenu (plans esthétique et formel) et, d'autre part, leurs fonctions, avec une attention particulière au rôle qu'ils jouent dans la promotion et la médiation de la musique de création (plans sociologique, pédagogique, communicationnel). Pour ce faire, nous nous appuyons sur les résultats de l'analyse exploratoire d'un corpus de 79 sites 
Internet de compositeur-rices joués dans l'édition 2017 de trois importants festivals internationaux de musique "contemporaine " (Musica de Strasbourg, La Biennale Musica de Venise et Montréal/Nouvelles Musiques). Nous déléguons donc à des institutions le choix des compositeur-rices dont nous étudions les sites Internet, ce qui introduit dès le départ un biais (les festivals pouvant rentrer dans la catégorie d' " institutions fortes " que la " révolution digitale " serait en train de supplanter). Ce choix offre néanmoins des avantages : ces festivals présentent des œuvres de jeunes artistes aussi bien que de compositeur rices entré·es dans le canon de la musique du second $\mathrm{xx}^{\mathrm{e}}$ siècle et ouvre sur une sélection très variée de compositeur-rices par âge, genre, provenance, formation et carrière. Ces trois festivals ont permis de constituer un corpus de 102 noms de compositrices (16) et compositeurs (86) vivants ou décédés après l'année $2000^{2}$, un nombre élevé grâce au bas niveau de redondances dans la programmation des festivals (un seul compositeur, Toshio Hosokawa, a été joué dans deux festivals). Nous y retrouvons un certain nombre de compositeurs décédés et désormais canoniques (Ligeti, Stockhausen, Xenakis, etc.) et de véritables vedettes internationales (de Thomas Adès à Tan Dun, de John Zorn à Helmut Lachenmann, etc.), ainsi que des compositeur.rices avec une importante carrière internationale, souvent reconnu·es comme des maîtres (de Sylvano Bussotti à Philippe Manoury) et beaucoup de jeunes parfois débutant·es (Clara Olivares et Ole Hübner, les benjamins du corpus, sont nés en 1993 - la moyenne d'âge des compositeur·rices considéré·es est cependant assez élevée, 55 ans).

\begin{tabular}{|c|c|c|c|c|c|}
\hline & $\begin{array}{c}40 \text { ans ou } \\
\text { moins }\end{array}$ & $\begin{array}{c}41 \text { ans ou } \\
\text { plus }\end{array}$ & Décédé & $\begin{array}{c}\text { Âge } \\
\text { inconnu }\end{array}$ & Total \\
\hline Pas de page personnelle & 4 & 14 & 3 & 2 & 23 \\
\hline Page personnelle & 24 & 47 & 4 & 4 & 79 \\
\hline Site personnel & 18 & 44 & & 4 & 66 \\
\hline $\begin{array}{l}\text { Pas de site personnel, } \\
\text { mais page SoundCloud. }\end{array}$ & 6 & 3 & & & 9 \\
\hline Site Internet « Hommage» & & & 4 & & 4 \\
\hline Total & 28 & 61 & 7 & 6 & 102 \\
\hline
\end{tabular}

Figure 1 : Type de page personnelle selon l'âge des compositeur rices.

2 Ce terminus post quem a été choisi arbitrairement et permet d'embrasser ces 20 dernières années de la démocratisation de l'accès à Internet, qui s'accompagne d'une injonction à être présent sur le web. Nous avons exclu, par exemple, Franco Donatoni (mort en 2000). Les compositeurs décédés après 2000 et rentrant donc dans notre corpus sont au nombre de sept : Karlheinz Stockhausen (1928-2007), Iannis Xenakis (1922-2001), Henri Dutilleux (1916-2013), György Ligeti (1923-2006), Gilles Tremblay (1932-2017), John Weinzweig (1913-2006), Jacques Hétu (1938-2010). Quatre d'entre eux (Stockhausen, Xenakis, Dutilleux, Weinzweig) ont un site Internet actif géré par une association ou des particuliers. 
Diversité des dispositifs : un clivage générationnel?

Des sites Internet officiels, aux sites Internet " hommage " en passant par des pages d'artistes sur des sites collectifs de type SoundCloud, trois grandes catégories de pages personnelles de compositeur rices sur Internet ont été dépouillées durant cette enquête. Huit compositeur-rices sur dix sont présents sur le web avec une page personnelle, et ce, quel que soit leur âge (figure 1).

Pour autant, les types de formats choisis par les compositeur.rices de " 40 ans ou moins » et de " 41 et plus » ne sont tendanciellement pas les mêmes. Les compositeur-rices de plus de 40 ans, certainement influencés par les stratégies néolibérales de marketing des années 2000 préconisant de tels outils de communication ${ }^{3}$, optent majoritairement pour la construction de sites web dédiés. Les moins de 40 ans optent plus généralement pour des pages SoundCloud (parmi ceux qui ont une page SoundCloud, les trois quarts ont moins de 40 ans). Or, ces choix de supports ne sont pas anodins. L'architecture personnalisable à souhait des sites permettant le déploiement et la mise en relation de documents photographiques, vidéographiques, sonores et scripturaux, contraste avec la rigidité formelle des pages SoundCloud, presque exclusivement dédiées au dépôt et à l'écoute de fichiers sonores visant à rassembler et faire connaître le répertoire d'un artiste tout en offrant la possibilité au public de commenter les œuvres. Alors que la page SoundCloud de Sofia Avramidou (née en 1988) donne accès à sa biographie et à huit enregistrements de ses œuvres (voir Annexe, capture $1^{4}$ ), le site Internet de Philippe Manoury (né en 1952) se caractérise par une profusion d'entrées et de ramifications peuplées de photos, de citations, d'articles de journaux, etc. (voir Annexe, capture 2) D'un côté, la musique est proposée seule, sans commentaire du·e la compositeur·rice, et de l'autre elle s'enrichit par le texte, l'image, la vidéo.

La variété de la structure des sites à proprement parler invite donc à se demander quelles sont la place (physique) et l'importance accordées aux écrits des compositeur·rices sur ces supports numériques et quel est le type de rapport à l'œuvre et au·à la compositeur rice qui y est envisagé.

\section{LA LIGNE ÉDITORIALE DES SITES}

L'usage généralisé des nouvelles technologies de communication et d'information dont témoignent les compositeur rices ${ }^{5}$ en investissant l'espace médiatique numérique s'apparente à la « dynamique communicationnelle » (Jacobi 1997, p. 9) adoptée au tournant des années 2000 par les musées. Jacobi analyse cette dernière comme «la

3 L'investissement massif d'Internet par les compositeur-rices n'est pas sans lien avec l'évolution managériale du travail des artistes. Sur cette question, lire l'article d'Alexandre Robert dans ce numéro.

4 En annexe de l'article - sur le site de la Revue musicale OICRM -, un fichier PDF présente les captures d'écran des pages des sites Internet des compositeur rices dont il est question ici afin de les figer dans le temps où ils ont été analysés.

5 L'enquête montre également que les compositeur·rices sont présent.es sur Facebook avec une page personnelle ou officielle pour 36 d'entre eux. 
façon dont le musée clame à l'extérieur que le cœur de la machine bat encore, qu'il est bien vivant »(Jacobi 1997, p. 9). La structure des sites Internet construits ou investis par les compositeur-rices peut-elle nous informer sur la façon dont ces artistes cherchent à affirmer leur présence sur les scènes musicales internationales?

\section{Structure et contenu}

Ce travail permet d'explorer les stratégies de présentation de soi des compositeur rices, mais aussi de faire saillir les tendances éditoriales de sites se caractérisant par leur architecture hétérogène qui parfois changent du tout au tout. Dans les pages d'accueil des sites, l'univers du·e la compositeur-rice se déploie dans des entrées hiérarchisées sous forme d'onglets thématiques dont la moyenne se situe dans notre corpus à 5 onglets par site, mais qui peut s'élever à un nombre de 21 (figure 2).

\begin{tabular}{|l|c|}
\hline Nombre d'onglets & Nombre de sites \\
\hline Moins de 5 onglets & 17 \\
\hline De 5 à 9 onglets & 53 \\
\hline 10 onglets et plus & 7 \\
\hline Lien rompu & 2 \\
\hline Total général & 79 \\
\hline
\end{tabular}

Figure 2: Nombre d'onglets par site Internet de compositeur rice.

Alors que l'on sait que les pages d'accueil des sites sont une " carte de visite décisive pour leur bonne exploration » (Bonhomme et Stalder 2006 p. 12), le défi pour les internautes tient à la profusion des intitulés d'onglets qui se décline à l'échelle de notre corpus sous 113 formulations différentes et parfois inattendues (ex. : Food Blog ${ }^{6}$, Korean $^{7}$ ). Seul le site Internet de Georges Aperghis (né en 1945) prend acte du dénuement dans lequel sont laissés les usagers de ces sites (voir Annexe, capture 5). Une précaution éditoriale rédigée par les «tenanciers » du site s'affiche donc sur sa page d'accueil précisant en trois points l'utilité de ce site qui « informe sur le travail du compositeur ", répertorie, donne accès à ses œuvres, et permet, via un blogue, de se tenir au courant de l'actualité du·e la compositeur rice. Dans tous les autres cas, difficile de se repérer dans le système de classement vernaculaire adopté par chacun des compositeur·rices selon leurs besoins spécifiques ${ }^{8}$ (figure 3 ).

\footnotetext{
6 Sur le site d'Andrea Liberovici (voir Annexe, capture 3).

7 Sur le site d'Andy Elmer (voir Annexe, capture 4).

8 Il en va ainsi de Kaija Saariaho (née en 1952) qui archive sur son site et sous l'onglet « electronics » les logiciels qui permettront de réinterpréter ses œuvres (voir Annexe, capture 6).
} 


\begin{tabular}{|l|c|}
\hline Intitulé des onglets & Nombre de sites \\
\hline Biographie & 59 \\
\hline Catalogue des œuvres & 53 \\
\hline Calendrier/actualités & 44 \\
\hline Contacts & 40 \\
\hline Accueil & 33 \\
\hline Photographies & 25 \\
\hline Presse/média & 25 \\
\hline Musique/sons & 24 \\
\hline Discographie & 24 \\
\hline Écrits & 19 \\
\hline Vidéos & 19 \\
\hline Contacts & 12 \\
\hline
\end{tabular}

Figure 3 : Intitulés des onglets figurant sur les pages d'accueil des sites Internet des compositeur rices (seuls sont mentionnés les onglets rencontrés plus de 10 fois durant notre dépouillement).

Les onglets les plus communs renvoient donc aux biographies des compositeur-rices, au catalogue de leurs œuvres, à leur calendrier de concerts et à leurs coordonnées. À l'inverse, une place plus que relative est accordée aux " écrits " des compositeur-rices sur ces pages d'accueil. Un onglet ne leur est dédié que dans un quart des cas et on a relevé une seule occurrence des onglets "note de programme ${ }^{9}$ " et " analyse musicale ${ }^{10}$ ». Il est donc légitime de se demander si ces sites sont des lieux "d'expression de » et de "familiarisation avec " la pensée et l'esthétique du·e la compositeur-rice ou plutôt une forme dématérialisée des outils promotionnels habituels calqués sur les attentes traditionnelles des institutions de concerts (notice biographique professionnelle, curriculum vitae, catalogue des œuvres, contacts des agents, etc.) - témoignage d'une certaine homéostasie du champ de la musique dominé par les pairs et les institutions.

Mise en vitrine des cuvres

La façon dont les œuvres des compositeur rices sont mises en vitrine sur leurs sites ajoute à la pertinence de cette question (cf. figure 4). En se contentant de mettre les usagers en contact avec les œuvres de façon directe (ex. : les vidéos de performance) ou différée (ex. : calendrier des prochains concerts), les créateur rices de ces sites semblent

9 Ceci est d'autant plus marquant que dans la page ouverte par cet onglet (voir Annexe, capture 7), Jukka Tiensuu (né en 1948) réfute en trois arguments clés l'utilité des telles notes de programme.

10 On trouve cet onglet sur le site de Manoury. Nous reviendrons sur le contenu de la page ouverte par cet onglet dans la section " Mise en vitrine des œuvres » de cet article. 
montrer qu'ils n'ont pas « fait le deuil du mythe de la révélation » (Donnat 2008) qui voudrait que la seule rencontre avec les œuvres suffise à produire un choc esthétique.

\begin{tabular}{|l|c|}
\hline Type de mise en vitrine des œuvres & Nombre de sites \\
\hline Streaming (intégral ou extraits) & 54 \\
\hline Vidéo YouTube d'une interprétation, parfois avec partition (extraits ou & 52 \\
\hline intégralité) & 26 \\
\hline Date des prochaines interprétations en concert & 23 \\
\hline Notes de programme présentant les œuvres & 13 \\
\hline Possibilité d'achat ou de location des partitions & 13 \\
\hline Visualisation des partitions et/ou téléchargement gratuit & 12 \\
\hline Extraits de partitions & 8 \\
\hline Possibilité d'achat des disques ou téléchargement payant des enregistrements & \\
\hline
\end{tabular}

Figure 4: Mise en vitrine des auvres sur les sites Internet des compositeur rices.

Le fait qu'on s'adresse à un public de pairs plutôt qu'au grand public trouve sa manifestation la plus radicale lorsque la présentation des ouvres repose sur la mise en ligne de leurs partitions dont l'audience se trouve alors réduite à celle des «lecteur.rices ». Les enregistrements d'œuvres ou leur streaming et les vidéos YouTube supplantent donc les formes explicatives, pédagogiques ou de vulgarisation telles que les notes de programmes (présentes sur seulement 23 sites), qui pourraient accompagner les internautes dans la découverte du travail du·e la compositeur·rice ${ }^{11}$. On pourrait penser que les compositeur-rices délèguent cette part de vulgarisation et de médiation de leur musique aux organismes de promotion de la musique de création, qui s'engagent effectivement de plus en plus dans cette voie (Kirchberg 2019). Des organismes comme l'Ircam ou le Portail de la musique contemporaine offrent respectivement des analyses en ligne mêlant texte, vidéo et extraits de partitions avec audio $^{12}$ et des cartes conceptuelles sur l'univers d'un œuvre ou d'un genre. Néanmoins, la production de ces outils n'est pas systématique (seulement 7 analyses et 11 cartes conceptuelles) et une seule personne de notre corpus (Kaija Saariaho) s'y trouve représentée. Dès lors, si des modèles existent bien, ils ne sont cependant pas encore pleinement exploités par le milieu, ni par les institutions, ni par les compositeur rices sur leur site personnel.

11 Pour une synthèse de la place des notes de programme dans la vulgarisation des œuvres musicales, voir Bernard 2019.

12 Sur cette page dédiée aux analyses d'œuvres, l'Ircam se donne la mission de " faire connaître les œuvres produites à l'Ircam à un public plus large ; montrer la relation entre l'idée musicale et les technologies utilisées ; identifier les nouveaux éléments du vocabulaire musical qui émergent à travers ces œuvres ; offrir un support d'information aux interprètes » (voir : http://brahms.ircam.fr/analyses/about/, page consultée le 9 décembre 2019). 
Seuls 17 compositeur·rices offrent la possibilité d'accéder à des analyses musicales disséminées sur leurs sites sans onglet dédié (cf. figure 5). Il faut cependant noter que ces analyses ne sont pas toujours consacrées aux œuvres présentes au catalogue du·e la compositeur·rice et que ce·tte dernier-ère n'en est pas toujours l'auteur·rice. Difficile alors de compter sur ces sites pour étayer, à coup sûr, sa connaissance du répertoire $\mathrm{du} \cdot \mathrm{e}$ la compositeur-rice. Sont-elles alors vraiment utiles aux programmateur rices ? Certains sites semblent vouloir offrir un espace plus personnel que la simple transposition web d'une carte de visite, mais deviennent ainsi un produit hybride duquel on peine à faire émerger une ligne éditoriale claire.

L'exemple du site de Manoury est à ce titre particulièrement représentatif de cette tension. Ce compositeur est le seul à consacrer un onglet aux " analyses musicales » (voir Annexe, capture 8). En décalage avec une logique promotionnelle, les écrits que met en valeur Manoury ne sont pas consacrés à l'analyse de sa propre production musicale, mais plutôt à l'analyse de compositions de Mozart, Berg ou Beethoven : le compositeur considère que ses écrits (y compris ses analyses d'œuvres d'autres compositeurs) font partie de sa production et méritent d'être médiatisés via le support numérique. En outre, aucune analyse ne figure sous l'onglet qu'il consacre à son opéra Kein Licht (voir Annexe, capture 9). Cet onglet ouvre en revanche sur une collecte de dons en ligne ${ }^{13}$ qui témoigne de la diversité des logiques d'usage et d' "exposition numérique de soi » (Granjon et Denouël 2010) attribuées à ces sites par les compositeur·rices.

\begin{tabular}{|l|r|}
\hline Analyses des œuvres sur le site ? & Nombre \\
\hline Pas d'analyses d'œuvres sur le site & 60 \\
\hline Présence d'analyse d'œuvre sur le site & 17 \\
\hline Écrites par le·a compositeur·rice & 9 \\
\hline Écrites par d'autres personnes & 5 \\
\hline Écrites par le·a compositeur·rice et par d'autres & 3 \\
\hline Lien rompu vers le site & 2 \\
\hline Total & 79 \\
\hline
\end{tabular}

Figure 5: Auteur-rices des analyses d'œuvres archivées sur les sites Internet des compositeur-rices.

Au terme de ce panorama de la facture des sites de compositeur rices, nous pouvons conclure que si nous sommes loin d'une médiation de ces œuvres contemporaines qui permette aux auditeurs-internautes usagers d'accéder aux codes, à l'esthétique et à la démarche compositionnelle de leurs auteur rices, nous ne sommes pas non plus dans une transposition à l'identique d'un modèle de communication institutionnelle établi. La place que les compositeur·rices accordent à leurs écrits dans les sites vient étayer cette remarque.

13 Philippe Manoury est à l'époque le premier compositeur en France à adopter le modèle économique $\mathrm{du}$ « financement participatif » pour soutenir la création de cet opéra. 
LES ÉCRITS DANS LES SITES

La "découvrabilité » des textes écrits par les compositeur·rices et déposés sur ces sites, soit le potentiel de ces écrits à capter l'attention d'un internaute (Desjardins 2016), est limitée. Seulement 16 compositeur-rices permettent d'accéder directement à leurs " écrits » en proposant un onglet dédié sur la page d'accueil. En outre, ces écrits sont de nature très différente (interviews données à des journalistes et des musicologues, textes de présentation des œuvres, blogue ou travaux universitaires ${ }^{14}$ ) et ne sont pas toujours accessibles en ligne.

L'hétérogénéité des documents ainsi rassemblés sous l'onglet «écrits » reflète celle, plus générale, des écrits de compositeur-rices disséminés à travers les sites. Plus de 29 types d'écrits ont été recensés lors de notre dépouillement. Les sujets disparates alors abordés par les compositeur-rices vont de résumés de romans ${ }^{15}$ à des textes poétiques ${ }^{16}$. Face à la diversité de la nature des écrits et de leur statut, nous concentrerons nos prochaines analyses sur les biographies et les notes de programme qui sont, pour les unes comme pour les autres, pratiquement toujours présentes sur ces sites.

\section{Les biographies de compositeur rices}

L'importance des biographies sur les sites de compositeur-rices se mesure à leur caractère presque incontournable sur ces plateformes (figure 6) : 59 sites leur consacrent un onglet spécifique (cf. supra figure 3), selon ce qu'on comprend être une exigence minimale compte tenu de son utilité pour les programmateurs et les médias. La destination de ces textes est révélée par leur format, qui ne s'éloigne que rarement de la forme standard de la notice biographique académique sans explorer de façons alternatives tirant parti de la nature du média numérique. Bien que majoritairement non signées, ces biographies sont souvent accompagnées d'un copyright du·e la compositeur $\cdot$ rice $^{17}$. Cette absence d'identification des auteur.rices des biographies (ou l'effacement volontaire de l'auctorialité des compositeur-rices ?) relève d'une stratégie éprouvée qui permet de conférer à ce genre de texte un statut d'objectivité que leur fond et leur forme vont intensifier.

\footnotetext{
14 Sur le site de Carlos Lopez Charles, par exemple (voir Annexe, capture 10).

15 Sur le site de Nicolas Gilbert (voir Annexe, capture 11).

16 Sur le site de Tolga Tüzün (voir Annexe, capture 12).

17 On trouve généralement en bas des sites une mention similaire à celle-ci : "All material copyright $($ C [nom du·e la compositeur·rice, année]».
} 


\begin{tabular}{|c|c|}
\hline Présence d'une biographie? & Nombre \\
\hline Lien rompu vers le site & 2 \\
\hline Non & 8 \\
\hline Oui & 69 \\
\hline Signée par le a compositeur rice & 2 \\
\hline Anonyme & 60 \\
\hline Signée par un tiers & 7 \\
\hline Total & 79 \\
\hline
\end{tabular}

Figure 6: Présence d'un onglet dédié aux biographies des compositeur.rices sur leurs sites Internet.

Ce constat se confirme à la lecture des deux seules biographies signées par les compositeurs eux-mêmes et rédigées à la première personne, par Benoit Montambault (né en 1984) et Iannis Xenakis (1922-2001). La première use presque à contreemploi du «je » dans une biographie volontairement allusive, qui n'informe pas sur le parcours du compositeur, mais est plus proche d'un texte poétique.

J'aimerais d'abord commencer en faisant appel à des parenthèses afin de les remplir avec n'importe quelle idée s'aventurant dans le coin : après tout, cela n'est-il pas leur rôle ? [...] D'une certaine façon, je crois que c'est ce que j'ai fait ces dernières années, j'ai cherché un peu partout des idées à insérer à l'intérieur de parenthèses, un peu comme on pourrait chasser des papillons à l'aide d'un filet (voir Annexe, capture 13).

La seconde autobiographie, signée par Xenakis et déposée par les administrateurs sur son site " hommage " (voir Annexe, capture 14), reprend pour sa part tous les poncifs d'une " entrée dans la musique " mue par une "nécessité intérieure "et racontée sur le mode vocationnel (Sapiro 2007 ; Pégourdie 2017). Les stratégies d'autoprésentation mises en mots par les compositeur-rices dans cet exercice, presque imposé, d' « écriture de soi », retiendront notre attention dans l'analyse des 60 autres biographies de compositeur rices non signées ou sous copyright.

1. Formes énumératives et délégation du discours : objectiver des parcours professionnels?

La rationalisation de la mise en page des biographies contribue à objectiver le parcours du·e la compositeur.rice ou son succès en accentuant l'aspect factuel de ces écrits. Thomas Adès (né en 1971) accole une frise chronologique des éléments marquants de sa vie de musicien à sa biographie rédigée (voir Annexe, capture 15). Que dire alors d'une telle entrée de la ligne du temps biographique : «1997-1999: Studies composition with Heiner Goebbels and Wolfgang Rihm in Karlsruh ${ }^{18}$ ". Ces informations, réduites à leur plus simple expression, s'adressent à un public aguerri ou constitué de

18 Sur le site de Jörg Widmann (voir Annexe, capture 16). 
professionnel-les (journalistes, directeur-rices d'institutions, etc.) qui sauront saisir l'intérêt musical de ces successions de dates, d'institutions et de noms propres et les mettre en récit. Dans le même esprit, les collaborateur rices d'Andrea Liberovici (né en 1962) relégué·es à droite de la biographie sont réduits à une énumération de noms égrainés comme autant d'attributs accumulés au profit de l'aura du compositeur (voir Annexe, capture 17).

Ces listes infrapaginales ne sont qu'une manifestation d'un trait stylistique commun à ces biographies qui comptent, dans leur grande majorité, de longs paragraphes énumératifs. La biographie d'Ana Sokolović (née en 1968) compte plus de 20 noms d'organisations et d'ensembles regroupés dans un seul paragraphe (voir Annexe, capture 18). Un habile jeu de virgules et de parenthèses permet même à Bruno Mantovani (né en 1974) d'agencer un emboitement de listes d'interprètes, de chefs et d'ensembles déclinés en noms propres ou d'institutions dont la lecture devient rapidement fastidieuse (voir Annexe, capture 19). Si l'on peut douter de l'efficacité communicationnelle de telles énumérations, on peut également s'interroger sur l'efficacité symbolique de cette stratégie itérative adoptée sans distinction par l'ensemble des compositeur-rices. La mise en vis-à-vis des références mobilisées par Raffaele Sargenti (né en 1980), en début de carrière, et Steve Reich (né en 1936), est à ce titre assez évocatrice. Là où le premier cite le « Festival 5 giornate (Milano, Italy)» ou le "Thailand International Composition Festival (Bangkok) " ${ }^{19}$ à la renommée relative dans le monde de la musique de création, le second, riche de sa carrière avancée, renvoie à des articles qui lui sont consacrés dans The Times ou The New Yorker $^{20}$. Reich couple donc cette modalité des listes à une stratégie de délégation d'autorité quant à la valeur de sa carrière de compositeur ("The Times dit », "The New Yorker dit $")^{21}$.

Ni vulgarisation d'un itinéraire de compositeur·rice et encore moins médiation d'un processus créateur, ces « esquisses autobiographiques » ressemblent plutôt à des «notices utilitaires » dans lesquelles la force cumulative de faits désincarnés vient lester le récit de

19 "His instrumental works are regularly performed and selected in several contemporary music festivals and call for scores, as Accademia di Santa Cecilia (Rome, Italy), Festival 5 giornate (Milano, Italy), GAMo concerti (Firenze, Italy), Sagra Musicale Umbra (Perugia, Italy), Musica Insieme Contemporanea (Bologna, Italy), Biennale di Venezia (Italy), iN Festival 2015 (Seoul, South Corea), Thailand International Composition Festival (Bangkok)» (http://raffsarge.wixsite.com/raffaelesargenti/bio, consulté le 9 décembre 2019 ; voir aussi Annexe, capture 20).

20 "Steve Reich was recently called "our greatest living composer" (The New York Times), "America's greatest living composer" (The Village VOICE), "...the most original musical thinker of our time” (The New Yorker) and "... among the great composers of the century" (The New York Times). The Swedish Academy said: "... Steve Reich has transferred questions of faith, society and philosophy into a hypnotic sounding music that has inspired musicians and composers of all genres" (voir https://www.stevereich.com/; voir aussi Annexe, capture 21).

21 Kenji Sakai (né en 1977), dont la carrière est beaucoup moins affirmée, use de la même stratégie et ne présente son travail qu'au prisme de ce qu'Helmut Lachenmann aurait dit de lui (la citation de Lachenmann n'est pas référencée) : "Helmut Lachenmann reconnaît en ce jeune compositeur une parfaite maîtrise compositionnelle et une grande virtuosité dans la gestion du son et du temps. Sa musique, imprégnée de clarté et de luminosité, développe un style hautement personnel qui aborde, sans complexe, une certaine forme de légèreté » (http://kenjisakai.net ; voir aussi Annexe, capture 22). 
carrière des artistes d'un poids symbolique dont la compréhension semble réservée aux initié es. Les destinataires de ces textes semblent donc être des professionnel·les (agent·es, programmateur-rices, chargé·es de communication) et, partiellement, des collègues (les énumérations créant alors un effet de compétition). Les biographies répondent donc au rôle promotionnel du site selon une approche traditionnelle et très institutionnalisée ; elles ne sont pas des agentes de vulgarisation et de médiation, et ne contribuent donc pas aux changements de dynamiques de présentation de soi et de son œuvre que pouvait laisser présager la plus grande liberté du régime numérique.

2. Récits désincarnés de «créateurs incréés »?

Si les stratégies de mises en page, d'énumération et de délégation d'autorité relevées dans ces textes rédigés à la troisième personne visent à éviter de tomber dans l'ornière d'un récit biographique réalisé dans un régime vocationnel, que nous disent ces biographies des carrières des artistes ? La description des activités non professionnelles des compositeur rices et de leur personnalité n'intervient que très rarement dans les biographies (cf. figure 7). Sept compositeur rices seulement se prêtent à l'exercice qui nous permet d'apprendre que George Crumb (né en 1929) possède " a shy, yet warmly eloquent personality" (voir Annexe, capture 23) et que le jeune compositeur canadien Joseph Glaser compose avec un œil de chorégraphe, car il « a pratiqué la danse pendant de nombreuses années " (voir Annexe, capture 24).

\begin{tabular}{|l|c|c|}
\hline Contenu de la biographie & Présent & Absent \\
\hline Formation musicale du/de la compositeur·rice & 12 & 50 \\
\hline État civil du/de la compositeur·rice & 19 & 43 \\
\hline Courants esthétiques de rattachement & 34 & 28 \\
\hline Vie non professionnelle du/de la compositeur·rice & 55 & 7 \\
\hline
\end{tabular}

Figure 7: Contenu de la biographie des compositeur ices présente sur leur site Internet.

Si les informations concernant l'état civil du·e la compositeur-rice, sa date de naissance et son pays d'origine sont présentes dans deux tiers des biographies, ces textes semblent plus majoritairement nous présenter les compositeur rices comme des « créateurs incréés » (Bourdieu [1981]2002), nés puis diplômés de conservatoires de renoms et programmés par des institutions musicales prestigieuses ${ }^{22}$. Au terme de la lecture des 50 des 62 biographies qui évoquent les étapes de la formation musicale

22 Ainsi, en va-t-il dans la biographie de Bruno Mantovani : « né le 8 octobre 1974. Après avoir remporté cinq premiers prix au Conservatoire national supérieur de musique et de danse de Paris (analyse, esthétique, orchestration, composition, histoire de la musique) et participé au cursus d'informatique musicale de l'Ircam, il débute une carrière internationale, et ses œuvres sont jouées au Concertgebouw d'Amsterdam, à la Philharmonie de Berlin, à la Philharmonie de Cologne, au Barbican center de Londres, au KKL de Lucerne, à la Scala de Milan, au Carnegie Hall et au Lincoln Center à New York, à la salle Pleyel à Paris et au Musikverein de Vienne » (http://www.brunomantovani.com/fr/biographie.html, nous soulignons ; voir aussi Annexe, capture 19). 
par les compositeur·rices, la question posée par Bourdieu reste généralement entière : mais qui a créé ces créateur rices? La réponse trouvée dans ces textes biographiques se résume alors souvent à la mention d'institutions musicales prestigieuses sans que l'on puisse savoir quels enseignants en composition ont marqué le parcours de ces artistes. Cela est d'autant plus étonnant que leurs professeurs de direction d'orchestre ${ }^{23}$ ou d'orchestration ${ }^{24}$ ont la part belle dans ces textes. En outre, la biographie des compositeur-rices ne permet pas systématiquement de mieux comprendre leur démarche artistique ni les courants musicaux dans lesquels ils se situent.

Près d'une fois sur deux, aucun repère (noms d'œuvres, de compositeur·rices marquant·es ou de courants esthétiques) n'est mentionné dans ces biographies. Plus encore, lorsqu'un discours sur l'esthétique du·e la compositeur-rice est développé, le propos, confus ou hypertechniciste, n'est pas toujours compréhensible. Maurilio Cacciatore (né en 1981) explique ainsi que ses productions « explorent des solutions non codées, en combinaison avec une recherche sur les équipements, les algorithmes et matières musicales spécifiques » (voir Annexe, capture 26).

Bien qu'étant un passage obligé sur les sites, ces biographies ne permettent pas de se familiariser avec le parcours, les productions ou la poétique du·e la compositeur-rice ${ }^{25}$. Certain·es en détournent volontairement le propos. C'est le cas d'Alexander Chernyskov qui, sur sa page SoundCloud, tourne cet exercice imposé en dérision :

Alexander Chernyshkov died in 2063 by being stabbed down in the metro in paris, apparently because he was selling some forbidden records and other material. [...] He became famous for his artwork in 2021, when he connected a living cow to the web 3.0 and started mutating the cells of the cow by random rotistic processes. [...] In 2030 they started doing their " sociofascistic " actions in athens and kuala lumpur and got imprisoned for drinking zwickl in the öffentlichkeit (voir Annexe, capture 27, nous reproduisons la typographie originale).

Les textes de présentation d'ouvres : études de cas

Après la biographie, le type d'écrit qu'on retrouve le plus souvent dans les sites de compositeur rices est la présentation d'œuvres. Il s'agit dans la plupart des cas de courts textes dont on devine l'origine dans les notes descriptives que les sociétés de concerts ou les étiquettes discographiques demandent aux compositeur-rices lors d'une création ou d'un enregistrement. 56 sites sur 79 n'offrent pas de textes de présentation des œuvres (cf. supra figure 4) et ne donnent dans la plupart des cas que des informations factuelles (effectif, date et lieu de création, durée, etc.) parfois accompagnées d'un extrait sonore (parfois même l'enregistrement intégral, audio ou

23 Martin Matalon, né en 1958, cite son professeur de direction, Jacques-Louis Monod, mais ne mentionne pas son (ou ses) professeur(s) de composition.

24 C'est le cas d'Andy Emler (voir Annexe, capture 25).

25 La structure et le contenu des sites fait de nous les témoins de ce qui semble être un double mouvement de refus de la prise de parole publique et dévoilement de données personnelles, puisque 26 sites donnent accès aux coordonnées personnelles des compositeurs. 
vidéo). Le site fonctionne donc dans ces cas comme une banque d'extraits, souvent très peu user-friendly par sa structure excessivement compliquée ; d'autres plateformes comme SoundCloud sont alors beaucoup plus efficaces, et, en effet, 9 des 102 compositeur·rices n'utilisent que ce média (cf. supra figure 1).

Lorsqu'un souci de présentation de l'œuvre se manifeste, il faut remarquer que l'auteur-rice n'expérimente aucune autre forme que celle, standard, du texte de présentation et, en quelques cas, des présentations orales sous forme de vidéos. Jamais, dans le corpus considéré pour cette étude, on ne retrouve des analyses techniques des pièces par leur compositeur-rice ou des tentatives de médiation par des moyens multimédias comme celles présentées plus haut (il n'existe pas une écriture vidéo intégrée aux œuvres : on a d'un côté des explications verbales, de l'autre des captures de performances). Le site devient alors surtout un lieu d'archive pour les notes de programme. Comme l'a souligné Yves Balmer dans son étude des " textes d'escorte » (notes de programme ou de pochette de disque) d'Olivier Messiaen, par leur genèse fortement reliée à une œuvre, ces textes rentrent dans la catégorie genettienne de " paratexte auctorial ", tandis que par leur nature éditoriale ils font partie de cette " littérature grise " qui échappe au dépôt légal et dont les modalités d'archivation ne sont pas homogènes (Balmer 2013, p. 28-29 ; la notion de " texte d'escorte » est introduite à la p. 35). L'accumulation progressive, la mise en série de ces textes dans les sites de leurs auteur·rices contribue à les sortir de la zone " grise " qu'ils occupent traditionnellement : le site leur donne en quelque sorte un statut de recueil publié, accessible en ligne et constamment mis à jour. Cela facilite et justifie la tâche de les analyser en tant que corpus, et de comparer les corpus entre eux. Quelques cas permettant d'illustrer les tendances relevées au cours de notre dépouillement seront présentés ici, dans le but de comparer les approches au genre du texte de présentation d'œuvre.

\section{Tan Dun : la voix du "génie »}

Compositeur du star-system, surtout connu grâce à ses musiques de film, Tan Dun (né en 1957) ne s'occupe pas personnellement de son site. Dans chacune des sections qui constituent l'onglet "Compositions " ( Chamber \& Solo Music ", " Multimedia \& Orchestra ", "Music Ritual \& Performance ", "Opera », etc.), la " présence » du compositeur est tout de même mise de l'avant par une courte citation (de Tan Dun ou d'autres à propos de lui) en ouverture de la page, dont le restant est constitué par une mosaïque de photos-liens menant chacune vers une page dédiée à œuvre dont elles sont l'icône. 
Considérons le cas de la section « $\underline{\text { Organic Music } ~(f i g u r e ~ 8) . ~}$



Figure 8: Sous-section "Organic Music» de la section "Composition» du site de Tan Dun (http: / /tandun.com/ compositions /, consulté le 9 décembre 2019).

Le fait que cette étiquette ne corresponde à aucun genre traditionnel (à la différence de "Opera », " Oratorio \& Chorus », "Symphonic Works \& Concertos », " Chamber \& Solo Music ») pourrait inciter à croire qu'on y trouvera des textes explicatifs. En effet, la citation de Tan Dun choisie par les rédacteurs du site donne une explication spirituelle/biographique de ce genre ${ }^{26}$, ce qui est un remarquable souci de vulgarisation si on la compare aux citations choisies pour la section "Opera " (qui met de l'avant la position - pas très claire - de Tan Dun face à la tradition du genre) ou pour "Chamber \& Solo Music » (un extrait d'une critique du Washington Post qui encense une œuvre en particulier) :

26 Une section « Organic Music », qui retrace brièvement l'histoire des œuvres que Tan Dun a regroupées sous ce nom, se trouve aussi dans l'onglet « About ", constitué par une biographie du compositeur par Frank J. Oteri. Il est remarquable que les œuvres citées par Oteri ne correspondent pas à celles incluses dans la section « Organic Music » de l'onglet « Composition », et qu'il n'y ait pas d'hyperliens de renvoi entre les deux pages. 
Organic Music: "Organic music concerns both matter of everyday life and matters of the heart. These ideas find their origin in the animistic notion that material objects have spirits residing in them, an idea ever-present in the old village where I grew up in China. "

Opera: "To me, opera is drama... What drama? It's not just character and story development. It could also be a dialogue between real and surreal, between past, present and future lives. From an avant-garde point of view, it could come from contrasting different materials. To me, opera has a wider field of meaning than the nineteenth-century definition. "

Chamber \& Solo Music: "A valuable addition to the keyboard literature, Eight Memories in Watercolor is a crystallin set of miniatures that spins traditional Chinese melodies around a core of tonal Western music, to evoke a delicately perfumed, quasi-impressionist sound world. "

Les textes de présentation des quatre œuvres appartenant au genre de l'organic music ne suivent pas un modèle homogène : le court texte écrit à la troisième personne pour Water Music (voir Annexe, capture 28) contraste avec les textes plus développés (et 17 photos) choisis pour le Paper Concerto (voir Annexe, capture 29), mélange d'interviews, de pensées de Tan Dun et d'une longue présentation de l'œuvre par Joanna C. Lee ; la page consacrée au Water Concerto (voir Annexe, capture 30) reproduit des extraits d'une interview et inclut deux vidéos où David Cossin parle de l'œuvre, tandis que le «moi » du compositeur prédomine dans la présentation du Earth Concerto (voir Annexe, capture 31), avec un texte de Tan Dun à la première personne et deux vidéos où l'on voit respectivement le compositeur choisir les instruments pour cette œuvre "for ceramic percussion and orchestra " et parler de son œuvre. Trois pages sur quatre citent un extrait d'une critique élogieuse. Cet échantillon est représentatif de la politique de communication du site de Tan Dun, où le discours des autres (critiques, musicologues) présentant le compositeur comme un génie dialogue avec une forte présence de la voix de Tan Dun (courtes citations ou textes plus élaborés) parlant de sa vie et de ses conceptions :

What is water? Sometimes you feel water is the voice of birth, or rebirth. But now I feel water is like tears, tears of nature. Every time I travel around I feel it's very difficult to find clean water. Everywhere is polluted almost. So it makes me feel difficult to sing with my music...

To me, my early life, living with water, having fun with water, and playing ritualistic music with water, has become very inspiring (Tan Dun 2007).

2. Martin Smolka : la prolifération des approches

Le site du compositeur pragois Martin Smolka, né en 1959, offre pour chaque œuvre de son catalogue une fiche technique suivie la plupart du temps par une note de programme souvent en plusieurs langues ${ }^{27}$ et parfois par des comptes rendus. La note de programme est presque toujours signée par le compositeur, parfois par d'autres ; il arrive que les textes dans les différentes langues ne soient pas des traductions et que le

27 Voir à titre d'exemple, celle pour Hubda hudbička/Music Sweet Music, en tchèque, polonais, anglais, allemand et slovène (voir Annexe, capture 32). 
compositeur ne soit l'auteur que d'une des versions, comme dans le cas de Lay, wail, purr, whirr, smooth, whoop, soothe (and this also is vanity), où Smolka signe un texte en tchèque, suivi par un texte en allemand de Stefan Fricke (voir Annexe, capture 33).

Le style des textes signés par Smolka n'est pas homogène et, généralement, il parle très peu de la musique qu'il a composée. L'œuvre est plutôt approchée par d'autres angles : le contexte autobiographique de sa conception (par exemple, Blue Bells or Bell $\underline{\text { Blues; }}$; voir Annexe, capture 34) des indications pour les interprètes (par exemple, Etudy pro sbor; voir Annexe, capture 35), ou encore les descriptions du contenu extramusical (par exemple, Psalmus 114) qui, là où elles ne se limitent qu'à exposer un programme, offrent au compositeur un moyen pour guider l'écoute par associations, comme dans le cas de Soutěska (In the Gorge):

Une Gorge (au sud de la Crète) : un endroit calme, loin du bruit du monde, la majesté des rochers, l'odeur de la pierre, les cris des oiseaux haut dans la petite bande bleue du ciel, les échos.

Mais la liberté des oiseaux là-haut est inaccessible, le fond impraticable ; en fait on se sent serré, angoissé, et derrière le tournant peut émerger un bloc qu'on n'aura ni assez de force ni assez de courage pour escalader.

Je trouve une semblable expression double dans les harmoniques naturals [sic] perchés dans l'aigu sur cordes graves du violoncelle. De délicats sons de velours continuent de résonner longuement dans les espaces intérieurs de l'instrument, je ne peux me lasser des échos, là-bas, loin à l'intérieur (voir Annexe, capture 36).

Parmi les notes de programme pour a yell with misprints (voir Annexe, capture 37), on retrouve une sorte de court manifeste ("Neuf règles pour composer de la musique "), un texte qui ne s'applique donc pas qu'à l'œuvre à laquelle il est associé dans le site. Le fait d'être en ligne ne sort pas forcément un texte de sa zone " grise » et nonobstant leur apparente immédiateté, certains sites s'offrent aux utilisateurs (musicophiles ou chercheurs) comme des fonds d'archives non inventoriés, des assemblages de matériaux préexistants non uniformisés et sans ligne éditoriale ${ }^{28}$. De plus, par rapport à des archives papier, les sites présentent une forme de rigidité qui peut forcer la catégorisation d'un contenu, puisque le compositeur n'est pas forcément un informaticien capable de modifier la structure de son site et se limite parfois à remplir un gabarit programmé par d'autres.

3. Lucia Ronchetti : la personnalisation des catégories

Dans les phases de programmation, avant de devenir rigide, le site permet par contre la plus grande souplesse d'organisation du matériel, notamment dans le choix des onglets. Lucia Ronchetti (née en 1963) est autrice d'œuvres musicodramatiques qu'elle regroupe en quatre catégories : "Music Theatre ", "Choral Opera ", "Drammaturgie ", "Action Concert Pieces ». Le fait d'insérer une œuvre dans 
une de ces catégories est déjà un acte discursif et la souplesse du média permet à la compositrice de présenter ses œuvres comme elle veut : c'est une forme de libération des institutions qui commandent des genres et dictent des étiquettes, si on suit la thèse de Lehmann.

Pour chaque œuvre, Rochetti fournit une fiche technique et à partir de 2000 une présentation $^{29}$ (non signée) et parfois des textes écrits par d'autres et des photos. Les présentations couvrent un continuum qui va d'un (1) discours portant exclusivement des éléments extramusicaux (intrigue, personnages, etc.) à une (4) description de la forme musicale, en passant par (2) l'explication de la façon dont une idée extramusicale est devenue geste/idée/langage musical et (3) des indications sur les moyens d'interpréter extramusicalement (sémantiser) le musical. Un exemple de cette dernière attitude se trouve dans Lascia ch'io pianga (voir Annexe, capture 38) de 2016 :

The soprano is inside the open piano, representing the unknown woman, a foreign woman on the move toward a new country, crossing the sea on a tragic black boat (the piano controlled by the pianist). [...] The woman is also a water nymph, the dreamlike projection of our fragmentary information concerning the moving of people towards Europe.

La frontière entre (2) et (3) est souvent floue, parce qu'expliquer comment l'extramusical devient musical implique de diriger l'interprétation du musical vers l'extramusical, comme dans ce texte écrit pour Lacus timoris (voir Annexe, capture 39) de 2015 :

The solo timpani is conceived as an unsounded lake, a magic cauldron collecting the deepest resonances coming from the orchestra's body. [...] The performer's gesture will become like the lecture of a mirror, which he can hear through, to capture some melodic fragments connected with fear and despair.

4. Philippe Hurel, Ofer Pelz : dans 1'atelier du compositeur

Les textes de présentations d'œuvres publiés sur les sites des compositeurs Philippe Hurel (né en 1955) et Ofer Pelz (né en 1978) ont ceci en commun d'aborder les œuvres d'un point de vue autobiographique. Presque entièrement écrits à la première personne, ces textes vont donc constituer une sorte d'autobiographie artistique de leur auteur complétant et enrichissant ainsi les biographies publiées sur leur site, écrites à la troisième personne et très factuelles :

[Hurel, D'un Trait, pour violoncelle seul (2007) ; voir Annexe, capture 40 :] Ayant joué du violon dans mon enfance, j'ai toujours repoussé le moment d'écrire pour les cordes seules, comme si la peur d'aborder mon instrument reprenait corps lors du passage à l'écriture. Mais l'amitié fait des miracles et Alexis Descharmes n'a eu aucun mal à me convaincre d'écrire pour le violoncelle. 
[Pelz, Climax (or Elements) pour orchestre de chambre (2015-16) ; voir Annexe, capture 41 :]

Cette pièce joue avec l'idée d'un moment de climax à l'arrêt, ou autrement dit, un moment stagnant du sommet musical.

Comme matériau harmonique principal, j'ai choisi le premier accord de la pièce «Le Chaos » de Jean-Féry Rebel, pièce qui précède l'œuvre que j'ai composée lors du concert. La stagnation est créée par la répétition perpétuelle de la phrase initiale qui introduit "l'accord Chaos ». J'utilise la répétition de façon irrégulière, une notion que je nomme « répétition instable » ou « fausse répétition ». Ainsi, avec l'emploi de la répétition, je tente de rendre ma musique plus perceptible, et de jouer à la fois avec l'attention et l'anticipation de l'auditeur, en introduisant des variations persistantes.

Voici que les compositeurs nous parlent de leurs fréquentations (amis musiciens) et nous font entrer dans leur laboratoire. Pelz nous renseigne sur les influences que le contexte d'exécution a pu exercer sur la composition de l'œuvre, donne des noms à des procédés techniques qu'il perçoit comme caractéristiques de son style et partage avec ses lecteur-rices le rapport qu'il souhaite établir entre sa musique et l'auditeur·rice. Bref, des éléments dont l'intérêt est loin d'être négligeable et qu'il faut avoir la chance de trouver en passant à travers les textes de présentation des œuvres ou les interviews et les articles que les compositeurs rendent accessibles sur leur site (mais cela reste exceptionnel, on parle d'une dizaine de cas dans notre corpus). Ces idées pourraient être autrement mises en exergue sur un site Internet - par exemple, comme le font les auteur.rices du site de Tan Dun, en isolant certaines citations éclairantes, ou encore en les regroupant dans un onglet dédié.
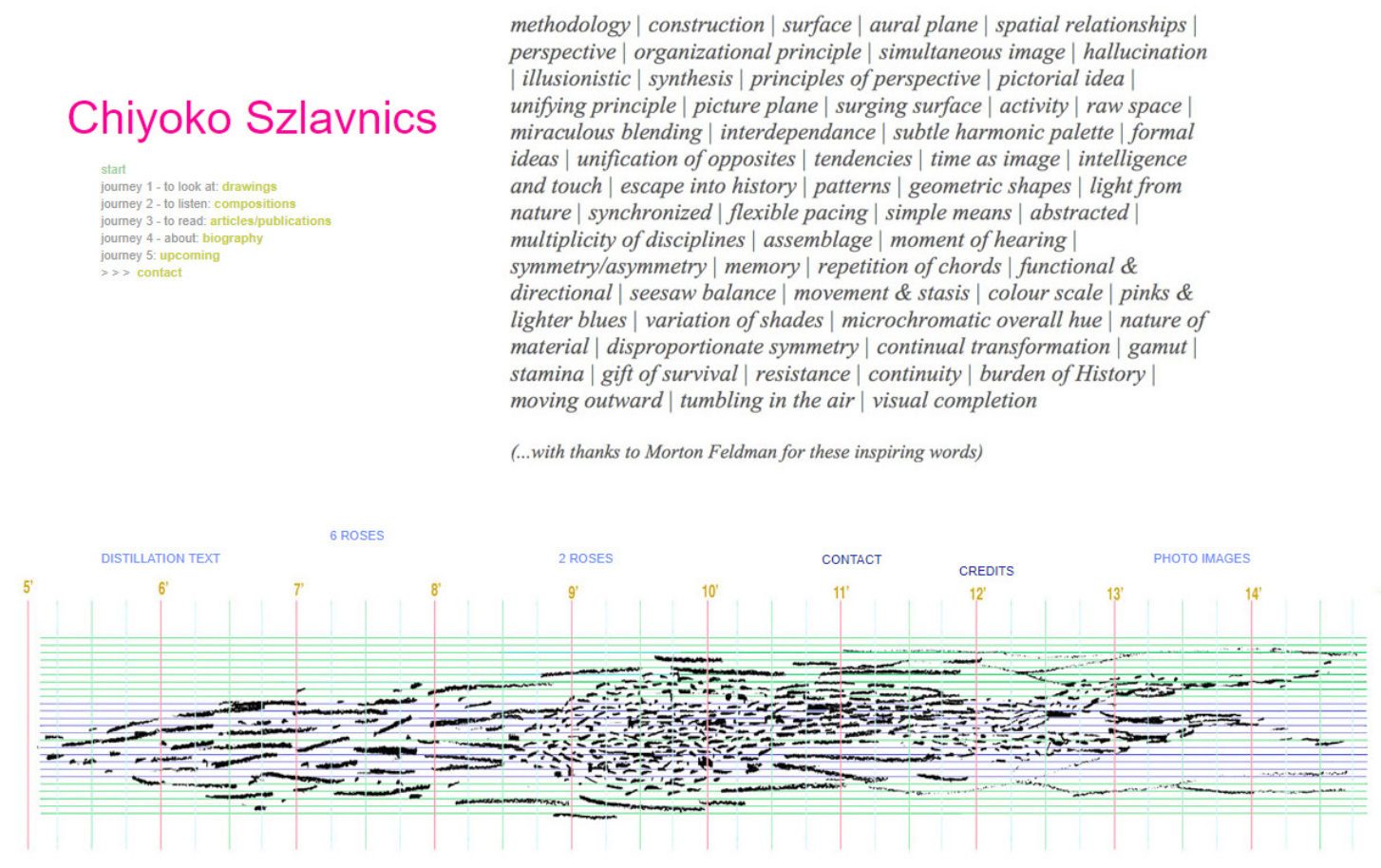

Figure 9: "Distillation text " dans le site de Chiyoko Szlavnics (http://www.chiyokoszlavnics.org, consulté le 9 décembre 2019). 
Cependant, fort peu nombreux sont les compositeur rices de notre corpus qui consacrent une place spécifique de leur site à l'expression de leur poétique et de leurs idées sur la musique (ou sur d'autres sujets). À partir de la page d'accueil du site de la compositrice Chiyoko Szlavnics, l'onglet « Distillation text » ouvre un texte/image (figure 9) qui est un condensé de l'article de Morton Feldman, "Between Categories» ([1969]1988) - une poétique à laquelle la compositrice souscrit donc pleinement (figure 9).

Manoury divise pour sa part ses écrits en catégories ("Sur mes œuvres »; " Esthétique »; "Sur les compositeurs »; "Sur la musique électronique » ; "Fragments du Japon "; voir Annexe, capture 42) ; en plus, son site contient une section "blog » intitulée Alla breve, où le compositeur publie des billets dans lesquels il exprime ses idées, notamment en réaction à des articles et à des livres (voir Annexe, capture 43). Le site de Smolka est représentatif, quant à lui, de la façon dont la poétique du compositeur peut être dispersée dans le site : nous avons déjà mentionné qu'un court manifeste sert de note de programme à a yell with misprints; à celui-ci s'ajoutent une exposition de la poétique du compositeur dans sa biographie (écrite par une autre personne, Petr Bakla) et un texte intitulé Manifesty listé parmi les écrits publiés du compositeur (voir Annexe, capture 44), mais non accessible via le site. Seulement deux compositeurs de notre corpus dédient un onglet à l'expression de leur poétique musicale, Liberovici (qui promeut en " manifeste " un extrait d'une interview ; voir Annexe, capture 45) ${ }^{30}$ et Jean Lesage (né en 1958), qui nourrit l'onglet " Poétique musicale " avec un très long texte structuré en sections, dont l'origine n'est pas déclarée (voir Annexe, capture 46).

Si les blogues de Manoury et Liberovici sont intégrés au site, il existe d'autres modalités d'existence de ces espaces d'expression. Jean-Louis Agobet (né en 1968) possède ainsi un blogue indépendant de son site (voir Annexe, capture 47).

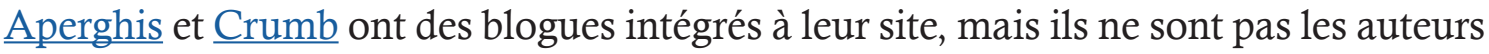
des billets (voir Annexe, captures 48 et 49). Le jeune compositeur Ole Hübner a créé un photo-blogue sur lequel il poste des photos de voyage (voir Annexe, capture 50) : dans ce cas aussi, donc, comme chez Hurel et Pelz, il s'agit d'une sorte de complément personnel à une biographie professionnelle écrite à la troisième personne (voir Annexe, capture 51).

\section{CONCLUSION : ÉCRIT/VISUALISATION, MÉDIATION/ARCHIVE}

Le site en soi comme forme d'écriture multimédia, le site comme lieu de création et de partage des écrits (y compris les « écrits » audiovisuels, comme les présentations vidéo, les conférences, les entrevues ${ }^{31}$ ), le site comme archive : comment ce

\footnotetext{
30 Son site présente aussi deux blogues, " Music Blog » et " Food Blog ». Ce dernier s'avère être inexistant.

31 Les entrevues figurent traditionnellement parmi les écrits d'un artiste : le site permet de les garder dans leur forme d'origine plutôt que de les transcrire. Similairement, la capture vidéo d'une conférence (nous avons, dans notre corpus, le cas d'une conférence TEDx par Joseph Glaser ; voir Annexe, capture 52) a un statut performatif et une composante multimédia qui se perdent à la simple lecture du texte.
} 
statut polyvalent du site exerce-t-il des fonctions de promotion, de vulgarisation et de médiation de la musique de son auteur-rice?

Lehmann (et on revient ainsi aux questions de "révolution digitale " discutées en introduction) clame que la révolution digitale ne se limite pas à "décomposer » le dispositif traditionnel de la " Nouvelle musique » du $\mathrm{xx}^{\mathrm{e}}$ siècle en en brisant l'institutionnalisation, mais aussi en confrontant l'idéal de « musique absolue » soutenu par ce dispositif traditionnel, centré sur le matériau musical et son exploitation méthodique. La mise en relation du matériau musical avec un concept et plus généralement avec le monde réel (son degré d' "iconicité ») serait, en revanche, la caractéristique qui permettrait à la musique de création de survivre dans l'univers digital où règne la loi quantitative. Selon cette perspective, il serait donc nécessaire, nous en concluons, que les compositeur rices favorisent le succès de leurs œuvres par une explicitation la plus immédiate possible de leur " concept». Lehmann distingue " trois stratégies de conceptualisation dans la musique contemporaine : la visualisation, la théâtralisation et la sémantisation ", pour remarquer que " c'est sans aucun doute la visualisation qui, à l'heure actuelle, influe le plus durablement sur la transmission des concepts musicaux » (Lehmann 2017, p. 180). La visualisation pourrait donc servir (et sert déjà dans plusieurs cas) à expliquer l'œuvre ${ }^{32}$, et ce, de façon beaucoup plus immédiate que la présentation écrite traditionnelle (dont la lecture n'est pas contemporaine à l'écoute). Pourtant, remarque Lehmann, " l'utilisation de la vidéo en musique contemporaine n'en est qu'à ses balbutiements et représentera sans aucun doute à l'avenir l'une des stratégies de conceptualisation les plus fortes. Sa fonction principale ne consistera probablement pas à visualiser une performance, mais à la documenter » (ibid., p. 181). Par « documenter », Lehmann entend " expliquer », rendre directement perceptible le processus créatif ou la sémantique de l'œuvre (il donne l'exemple de $\underline{\text { HardBeat de }}$ Leah Muir (2009) ; ibid., p. 182-183).

Pourtant, dans les sites que nous avons examinés, les compositeur· rices n'exploitent pas le potentiel du multimédia pour favoriser une écoute guidée de l'œuvre en temps réel. Nous avons seulement trouvé deux cas de vidéos comportant une partition déroulante et quelques cas où la prise de vue rapprochée d'une performance et le montage aident à relier action instrumentale et résultat sonore de façon plus claire (et guidée) qu'on ne pourrait le faire dans une salle de concert (mais c'est une technique expérimentée dans la reprise vidéo des concerts symphoniques ; voir Varon 2011).

En somme, la possibilité que les compositeur rices ont finalement de présenter leur musique de façon non traditionnelle et captivante n'est généralement pas exploitée. En d'autres mots, les compositeur rices ne semblent pas s'intéresser aux possibilités que le web leur offrirait pour présenter leur musique à un plus large public, et les sites, dans leur variété de formes, ne semblent pas s'éloigner généralement du modèle traditionnel du portfolio ${ }^{33}$. Ceci témoigne du fait que le monde de la musique de

32 Pour une série d'exemples de ce type, consulter le Répertoire raisonné de dispositifs de médiation de la musique constitué par les chercheurs de l'équipe Partenariat sur les publics de la musique.

33 On perçoit ainsi mieux la pertinence du Plan d'action pour la musique déployé par le ministère de la Culture et des Communications du Québec lancé en 2017 et qui vise à « outiller les créateurs et l'industrie 
création est finalement plus institutionnalisé que le laisse entendre l'essai de Lehmann. Serait-ce parce que, comme nous l'avons vu dans nos analyses des sites, la majorité des compositeur rices ne s'emparent pas encore pleinement du potentiel du numérique?

Archives, lieux d'expression, outils de promotion, les sites restent tout de même des endroits auxquels on ne parvient pas par hasard. Qui les visite ? Quelles sont les stratégies pour leur donner de la visibilité ? Ce sont des questions qui dépassent le cadre de la présente étude et qu'il faudra aborder en tenant compte des relations entre les sites et les réseaux sociaux sur lesquels les compositeur-rices sont actifs et actives et de la présence de liens vers les sites dans les plateformes de streaming comme SoundCloud ou dans les répertoires biobibliographiques en ligne ${ }^{34}$.

\section{BIBLIOGRAPHIE}

Balmer, Yves (2013), «Entre analyse et propagande. Olivier Messiaen et son usage des notes de programme ", dans Valérie Dufour, Marie-Hélène Benoit-Otis et Michel Duchesneau (dir.), Écrits de compositeurs. Une autorité en question, Paris, Vrin, p. 27-47.

Bernard, Justin (2019), Notes de programme. Une histoire, des pratiques et de nouveaux usages numériques, thèse de doctorat, Université de Montréal.

Bonhomme, Marc, et Pia Stalder (2006), " Analyse sémiolinguistique des pages d'accueil des sites politiques suisses sur Internet ", Mots. Les langages du politique, $\mathrm{n}^{\circ}$ 80, p. 11-23.

Bourdieu, Pierre ([1981]2002), " Mais qui a créé les créateurs ?", dans Questions de sociologie, Paris, Éditions de Minuit, p. 207-221.

Desjardins, Danielle (2016), "Découvrabilité. Vers un cadre de référence commun. Volet 1 ", Fond des médias du Canada, initialement publié sur le site https://decouvrabilité.ca/ (actuellement inactif), consultable sur https://docplayer.fr/28501928-Decouvrabilite-vers-un-cadre-de-referencecommun-volet-1.html, consulté le 9 décembre 2019.

Donnat, Olivier (2008), « Démocratisation de la culture. Fin... et suite ? », dans Jean-Pierre Saez (dir.), Culture et société. Un lien à recomposer, Toulouse, Éditions de 1'Attribut, p. 55-71.

Dorin, Stéphane (dir.) (2018), Déchiffrer les publics de la musique classique. Perspectives comparatives historique et sociologique/Unraveling Classical Music. Historical, Sociological and Comparative Perspectives, Paris, Éditions des Archives contemporaines.

Dujol, Lionel, et Silvère Mercier (2017), " Médiation numérique des savoirs et marketing public. Différences et complémentarités ", Documentation et bibliothèques, vol. 63, $\mathrm{n}^{\circ}$ 2, p. 5-11, https://doi.org/10.7202/1040175ar, consulté le 9 décembre 2019.

Feldman, Morton ([1969]1988), "Between Categories », Contemporary Music Review, vol. 2, n 2 («Listening 2 »), p. 1-5, https://doi.org/10.1080/07494468808567063, consulté le 9 décembre 2019.

Granjon, Fabien, et Julie Denouël (2010), «Exposition de soi et reconnaissance de singularités subjectives sur les sites de réseaux sociaux ", Sociologie, n 1, p. 25-43.

de la musique pour la maitrise du numérique » (http://culturenumerique.mcc.gouv.qc.ca/101-outiller-lescreateurs-et-lindustrie-de-la-musique-pour-la-maitrise-du-numerique/, consulté le 9 décembre 2019).

34 Voir par notamment la Base de documentation sur la musique contemporaine B.R.A.H.M.S. - Ircam, le Centre de musique canadienne ( $\mathrm{CMC}$ ), The Living Composers Project ou la Banca dati compositori italiani (BDCI) du Comitato nazionale italiano per la musica (СIDIM). 
Jacobi, Daniel (1997), «Les musées sont-ils condamnés à séduire toujours plus de visiteurs ?», La lettre de l'OCIM, n ${ }^{\circ} 49$, p. 9-14.

Kirchberg, Irina (2019), Panorama de la médiation de la musique au Québec. Définitions, acteurs et enjeux, Montréal, Partenariat sur les Publics de la Musique $\left(\mathrm{P}^{2} \mathrm{M}\right)$ et Conseil Québécois de la Musique (CQM), http://p2m.oicrm.org/wp-content/uploads/2019/07/V2-KIRCHBERG-Panorama-dela-me \%CC\%81diation-de-la-musique-au-Que\%CC\%81bec-FINAL-mars-2019-1.pdf, consulté le 9 décembre 2019.

Lehmann, Harry (2017), La révolution digitale dans la musique. Une philosophie de la musique, traduit de l'allemand par Martin Kaltenecker, Paris, Allia.

Menger, P[ierre]-M[ichel] ([1983]2001), Le paradoxe du musicien. Le compositeur, le mélomane et l'état dans la société contemporaine, nouv. éd., Paris, L'Harmattan.

Menger, Pierre-Michel (1986), "L'oreille spéculative. Consommation et perception de la musique contemporaine ", Revue française de sociologie, vol. 27, n 3, p. 445-479, https://doi.org/10.2307/3321318, consulté le 9 décembre 2019.

Menger, Pierre-Michel (2002), Portrait de l'artiste en travailleur. Métamorphoses du capitalisme, Paris, Seuil.

Pégourdie, Adrien (2017), " Devenir musicien "ordinaire". Construction et entretien des vocations des enseignants de musique classique », Bien symboliques/Symbolic Goods, $\mathrm{n}^{\circ} 1$ ("Artistes ordinaires/Ordinary Artists »), 15 octobre, https://revue.biens-symboliques.net/108, consulté le 9 décembre 2019.

Reibel, Emmanuel (2013), «Quel statut pour les écrits de compositeurs ? Éléments pour une réflexion méthodologique à travers l'exemple d'Hector Berlioz », dans Dufour, Benoit-Otis et Duchesneau (2013), p. 117-127.

Sapiro, Gisèle (2007), " La vocation artistique entre don et don de soi », Actes de la recherche en sciences sociales, vol. 168, n 3, p. 4-11.

Sinigaglia-Amadio, Sabrina, et Jérémie Sinigaglia (2017). Questions de culture. Temporalités du travail artistique: le cas des musicien.ne.set des plasticien.ne.s, p. 155-195. https:/ /www.cairn.info/temporalitesdu-travail-artistique-le-cas-des-musi--9782111515161.htm, consulté le 9 décembre 2019.

Tan Dun (2007), "Synopsis » [du Water Concerto for Water Percussion and Orchestra], tiré d'une entrevue avec Helen Elmquist, mai, http://tandun.com/composition/water-concerto-for-water-percussionand-orchestra/, consulté le 9 décembre 2019.

Varon, Gaia Valeria (2011), "Perfoming Performances. Some Considerations on the Role of the Filming and Recording Crews in Classical Music Videos ", communication au colloque international Analyser les processus de création musicale/Tracking the Creative Process in Music, Lille, 29 septembre-30 octobre, http://tcpm2011.meshs.fr/, consulté le 9 décembre 2019.

SITOGRAPHIE ${ }^{35}$

Institutions et banques de données

Banca dati compositori italiani (BDCI), http://www.cidim.it/cidim/content/109.

Base de documentation sur la musique contemporaine (B.R.A.H.M.s.), http://brahms.ircam.fr/.

Biennale musica 2017 (61. Festival internazionale di musica contemporanea), http://www.labiennale.org/it/ musica/2017.

Centre de musique canadienne, « Vitrine des compositeurs », https://www.musiccentre.ca/fr/compositeurs. 
Dicteco, dictionnaire des écrits de compositeurs, https://dicteco.huma-num.fr/.

Ministère de la Culture et des Communication du Québec, Plan d'action pour la musique, https://mcc.gouv.qc.ca/index.php?id=6124.

MNM, Montréal/Nouvelles musiques, $8^{e}$ édition, "Retour vers le futur », http://www.smcq.qc.ca/mnm/ $\underline{\mathrm{fr} / 2017 / .}$.

Musica Festival Strasbourg, édition 2017, https://festivalmusica.fr/documentation/editions/2017.

$P^{2} M$, Partenariat sur les publics de la musique, http://p2m.oicrm.org/.

Répertoire raisonné de dispositifs de médiation de la musique, http://mediationdelamusique.oicrm.org/.

The Living Composers Project, http://composers21.com/.

\section{Sites de compositeurs cités dans l'article}

Adès, Thomas, site personnel, http://thomasades.com/.

Aperghis, Georges, site personnel, http://www.aperghis.com/.

Avramidou, Sofia, page SoundCloud, https://soundcloud.com/sofia-avramidou-3/.

Cacciatore, Maurilio, site personnel, http://www.mauriliocacciatore.com/.

Chernyshkov, Alexander, page SoundCloud, https://soundcloud.com/alexander-chernyshkov.

Crumb, George, site personnel, http://www.georgecrumb.net/.

Elmer, Andy, site personnel, http://www.andyemler.eu/.

Gilber, Nicolas, site personnel, https://nicolasgilbert.ca.

Glaser, Joseph, site personnel, https://www.jglasermusic.com/.

Hübner, Ole, site personnel, https://olehuebner.wordpress.com.

Hurel, Philippe, site personnel, http://philippe-hurel.fr.

Liberovici, Andrea, site personnel, https://liberovici.it.

Lopez Charles, Carlos, site personnel, http://www.carloslopezcharles.com.

Mantovani, Bruno, site personnel, http://www.brunomantovani.com.

Manoury, Philippe, site personnel, http://www.philippemanoury.com/.

Matalon, Martin, site personnel, https://martinmatalon.com/.

Montambault, Benoit, site personnel, https://www.benoitmontambault.com.

Pelz, Ofer, site personnel, http://www.oferpelz.com/.

Reich, Steve, site personnel, https://www.stevereich.com/.

Ronchetti, Lucia, site personnel, http://www.luciaronchetti.com/.

Saariaho, Kaija, site personnel, http://saariaho.org/.

Sakai, Kenji, site personnel, http://kenjisakai.net.

Sargenti, Raffaele, site personnel, http://raffsarge.wixsite.com/raffaelesargenti.

Szlavnics, Chiyoko, site personnel, http://www.chiyokoszlavnics.org.

Smolka, Martin, site personnel, http://www.martinsmolka.com.

Sokolović, Ana, site personnel, http://www.anasokolovic.com/.

Tan Dun, site personnel, http://tandun.com/.

Tiensuu, Jukka, site personnel, https://tiensuu.fi. 
Tüzün, Tolga, site personnel, http://tolgatuzun.net/.

Widmann, Jörg, site personnel, https://www.joergwidmann.com. 